\title{
ENSAYAR CATEGORÍAS SOBRE GÉNERO Y SALUD: UN DESAFIOO NECESARIO
}

\section{Sofía Beatriz da Costa Marques ${ }^{1}$ \\ María Florencia Linardelli}

Resúmen: El campo del género y la salud es altamente heterogéneo y contiene una multiplicidad de preguntas, enfoques y problemáticas. A través de una revisión y análisis documental, el presente artículo presenta dos cuestiones centrales. Por un lado, la dificultad para agrupar investigaciones altamente diversas, contextos de producción de conocimiento muy desiguales y producciones movilizadas por problemáticas diferentes, con el consiguiente riesgo de presentar una falsa homogeneidad o de invisibilizar tensiones, conflictos y diferencias. Por otro, la potencia y el largo camino recorrido por investigadoras interesadas en la salud de las mujeres, las desigualdades sexo-genéricas, el control de los cuerpos femeninos/feminizados.

Palabras clave: género; salud; producción de conocimiento.

\begin{abstract}
The academic field of gender and health is heterogeneous and include a multiplicity of questions, approaches and problems. Through a documentary analysis and review, this article presents two central issues. On the one hand, the difficulty for grouping together highly diverse researches, contexts of production of knowledge very unequal and productions guided by different investigation problems. All these involve the risk of presenting a false homogeneity or make invisible tensions, conflicts and differences. On the other hand, we find the power and the long path walked by the researchers interested in women's health, gender inequality and control over feminine.
\end{abstract}

Keywords: gender; health; production of knowledge.

\section{Introducción}

Hablar de producciones académicas sobre "género y salud" implica remitirnos a un campo que alberga una diversidad tal que cualquier intento de clasificación y ordenamiento de los aportes afronta, en principio, el peligro de crear categorías al modo de la enciclopedia china que describe Borges en su cuento El idioma

\footnotetext{
1 Instituto de Ciencias Sociales, Humanas y Ambientales (INCIHUSA), CCT-CONICET Mendoza/Argentina. E-mail: sofiadacostamarques@gmail.com.

2 Instituto de Ciencias Sociales, Humanas y Ambientales (INCIHUSA), CCT-CONICET Mendoza/Argentina. E-mail: mlinardelli@mendoza-conicet.gob.ar.
} 
analítico de John Wilkins"3: "En sus remotas páginas está escrito que los animales se dividen en a) pertenecientes al Emperador b) embalsamados c) amaestrados d) lechones e) sirenas f) fabulosos $g$ ) perros sueltos $h$ ) incluidos en esta clasificación i) que se agitan como locos j) innumerables $k$ ) dibujados con un pincel finísimo de pelo de camello I) etcétera $m$ ) que acaban de romper el jarrón $n$ ) que de lejos parecen moscas". No obstante, si avanzamos en la lectura, el autor nos brinda algo de solaz: "La imposibilidad de penetrar el esquema divino del universo no puede, sin embargo, disuadirnos de planear esquemas humanos, aunque nos conste que éstos son provisorios".

Una vez advertidas del riesgo que las categorizaciones suponen, en este trabajo nos proponemos presentar algunos de los aportes de los estudios de género y feministas al campo de la salud ${ }^{4}$ que consideramos relevantes, con la finalidad de evidenciar la heterogeneidad de este campo y la diversidad de preguntas, enfoques y problemas que aborda. A lo largo del artículo se ponen de manifiesto dos cuestiones centrales. Por un lado, la ya mencionada dificultad para agrupar investigaciones altamente diversas, contextos de producción de conocimiento muy desiguales y producciones movilizadas por problemáticas diferentes, con el consiguiente riesgo de presentar una falsa homogeneidad o de invisibilizar tensiones, conflictos y diferencias. Por otro, la potencia y el largo camino recorrido por investigadoras interesadas en la salud de las mujeres, las desigualdades sexo-genéricas y el control de los cuerpos femeninos/feminizados.

De acuerdo al problema propuesto, el presente trabajo se basa en una revisión y análisis documental y se organiza en tres secciones: inicialmente realizamos una presentación del campo de los estudios feministas y de género, su contexto de surgimiento y algunas discusiones sobre la propia categoría de género. En segundo lugar, llevamos adelante un breve recorrido por algunas de las discusiones centrales acerca de lo que se entiende como campo de la salud. En tercer término, proponemos una categorización sui géneris de lo que consideramos los aportes y discusiones más relevantes que los estudios de género y los feminismos han suscitado en el terreno de la la salud/enfermedad. Es en este apartado donde recurrimos a una cierta imaginación categorial para organizar la diversidad de propuestas, enfoques y procedencias de las autoras. El primer grupo de trabajos se refiere a las intervenciones biomédicas sobre el cuerpo de las mujeres; el segundo, al androcentrismo y sesgos de género en la medicina; el tercero reúne estudios vinculados a enfermedades y padecimientos como experiencias marcadas sexualmente y el último aborda desarrollos acerca de la salud sexual y re-

\footnotetext{
3 Borges, Jorge Luis (1989). Otras Inquisiciones. En: Borges, Jorge Luis. Obras completas. T.2. Barcelona: Emecé. Este cuento aparece en el prefacio de Las palabras y las cosas (1966) donde Michel Foucault cita dicho texto con el fin de examinar el problema del orden, la clasificación y el lenguaje.
}

4

Nos circunscribimos a los estudios de género y feministas, sin desconocer la existencia de estudios de género

referidos a masculinidades, numerosos producidos desde el colectivo LGTTTBIQ, muchos de ellos enmarcados en los estudios queer que, no obstante su riqueza, superan los límites y objetivos de este artículo. 
productiva de las mujeres. Finalmente, y siguiendo el lema del último Encuentro Feminista de América Latina y el Caribe "diversas pero no dispersas", exponemos preocupaciones comunes sin desconocer las diversidades profundas de los estudios de género y feministas. Aquí enfrentamos el desafio de transitar un camino sinuoso que, a la vez que reconoce la existencia de puntos comunes, presenta tensiones entre las distintas perspectivas y los caminos bifurcados de las agendas de investigación de acuerdo a la localización socio-espacial de las investigadoras.

\section{Breves notas sobre los estudios de género y feministas}

Resulta forzado realizar una historia del surgimiento de los estudios de género y feministas. Más bien, lo que deviene posible es señalar algunos puntos geográficos y temporales relevantes para la construcción de este campo. Los primeros antecedentes pueden encontrarse en los denominados "estudios de la mujer", cuyo contexto de surgimiento se produce en los países centrales de Occidente a partir de la década de 1960 (BELLUCCI, 1992). Éstos fueron llevados adelante mayoritariamente por académicas que, desde posiciones de relevancia en sus distintas especialidades, comenzaron a analizar las diferentes facetas de la subordinación de las mujeres (FERNÁNDEZ, 2003). Además de aportar visibilidad a dicha situación, permitieron identificar la generalización de las situaciones de discriminación hacia las mujeres y cuestionar el androcentrismo del pensamiento científico.

En América Latina el desarrollo de los estudios de género y feministas se produce a finales de la década de los setenta y principios de los ochenta, ligado a una serie de procesos que caracterizaron a la región en ese momento histórico: el retorno de la democracia en gran parte de los países, la expansión de la matrícula femenina en educación media y universitaria, el desarrollo del movimiento de mujeres de la mano de otros movimientos sociales. Efectivamente, una de las características de los estudios de género en nuestras latitudes es que fueron precedidos por el movimiento de mujeres (LEÓN, 2004). Rastreando los primeros pasos de los feminismos del sur, Agüero (2015) cuestiona el mito que atribuye el origen de los feminismos latinoamericanos a la "importación" de ideas surgidas en los países del norte, desde donde se habrían expandido y difundido planteos producidos en las usinas de pensamiento de Europa y Estados Unidos sobre los/ las sujetos ubicados en otras latitudes. Crítica de este sentido común dominante sobre la historia de las ideas, la autora afirma que las reflexiones feministas en nuestras latitudes no se produjeron a partir de actos de recepción pasivos y transparentes "sino más bien como procesos de 'contaminación' y 'apropiación', producto del contacto de una idea -surgida en un determinado contexto de producción- con un horizonte de recepción heterónomo, el cual le otorga su impronta particular, transformándola, necesariamente, en otra idea" (2015, pág. 107). Respecto de los feminismos latinoamericanos Ciriza (2015) plantea que 
sus genealogías tienen múltiples y contradictorias raíces que localizadas tanto en las disímiles experiencias de explotación y dominación, como en las resistencias de mujeres y feministas en estas tierras. Puntualmente, en torno al surgimiento de los estudios de género en América Latina destaca: "El ciclo de conferencias promovidas por Naciones Unidas y la organización de los Encuentros Feministas Latinoamericanos y del Caribe, como también el papel de las mujeres en la resistencia a las dictaduras del cono Sur, a las políticas de exterminio en Centroamérica y a las políticas neoliberales de austeridad en todos nuestros países trajeron un interés creciente en los estudios de género e incluso en la teoría feminista en un contexto en el que, sin embargo, las desigualdades entre las mujeres no han dejado de aumentar" (CIRIZA, 2015, pág. 90).

Un nudo relevante en esta breve historización se ubica en los años setenta, cuando desde el feminismo anglosajón en los ámbitos académicos se impone el uso de la categoría gender (género), con el objeto de diferenciar las construcciones sociales de la biología. Estas académicas consideraban que la diferenciación entre sexo y género podía enfrentar mejor el determinismo biológico y ampliar los argumentos teóricos a favor de la igualdad de las mujeres (LAMAS, 1999). Aunque los primeros antecedentes de la noción de género pueden ser ubicados a mediados del siglo XX, cuando Simone de Beauvoir o Margaret Mead llamaron la atención sobre la construcción sociocultural de las diferencias sexuales (ESTEBAN, 2003), fue desde desde la psicología médica que se introdujo el término género a las ciencias sociales. Robert Stoller en Sex and Gender (1968) establece por primera vez con precisión la diferencia entre sexo y género, a partir del estudio de la identidad sexual en casos de niños y niñas cuyo sexo genético, anatómico y hormonal no resultaba coincidente. En dicho estudio se identificó el papel de la socialización como elemento clave en la adquisición de la identidad femenina o masculina, a partir de lo cual comienza a reservarse el uso del término "sexo" para aquellos aspectos fisiológicos y biológicos del ser macho o hembra, mientras que "género" comienza a nombrar las construcciones sociales existentes en torno a esas diferencias sexuales (GARCíA, 2006).

El uso de la categoría de género trascendió los límites del campo disciplinar en que surgió - psicología- y fue recuperada por otras ciencias sociales como antropología, sociología, economía, historia, entre otras. La aplicación a campos diversos amplió el sentido de un concepto que " [...] se fue definiendo en término de status, de atribución individual, de relación interpersonal, de estructura de la conciencia, como modo de organización social, como ideología o como simple efecto del lenguaje" (OSBORNE \& PETIT, 2008, pág. 148). Esta diversificación y complejidad adquirida por la noción suscitó algunos trabajos clave que revisaron su uso, los problemas que comportaba la imprecisión conceptual que había adquirido, las dificultades de traducción del término, al tiempo que propusieron conceptualizaciones específicas (RUBIN, 1986; SCOTT, 1986; LAMAS, 1999). La 
diversidad de usos y atribuciones dadas a la noción, no impidió que para autoras como Haraway (1991) el concepto de género sea considerado una herramienta analítica central de la perspectiva feminista. Asimismo, Moore (2009, pág. 9) indica: "La crítica feminista no se basa en el estudio de la mujer, sino en el análisis de las relaciones de género y del género como principio estructural de todas las sociedades humanas". Por su parte, Esteban (2003, pág. 23) sostiene que hablar de género supone hablar "de feminismo y desde el feminismo siendo este un espacio de saber y acción específico, aunque heterogéneo, que trabaja en pos del objetivo - más o menos explícito- de modificar las condiciones sociales que mantienen la subordinación de las mujeres".

Pero también otras autoras, como las provenientes del feminismo materialista francófono, descartaron su utilidad teórica e importancia explicativa, al tiempo que propusieron hablar de rapport sociaux de sexe (relaciones sociales de sexo) - bien de relaciones de sexaje (GUILLAUMIN, 2005 [1992]). Otro relevante espacio de crítica a la forma en que el feminismo de los paises centrales habría conceptualizado las relaciones de género provino de las feministas Negras estadounidenses, quienes señalaron la simultaneidad de las opresiones sexo-genéricas, raciales y de clase (DAVIS, 2005; COLLINS, 1998). Esta crítica fue acompañada por autoras de América Latina y el Caribe, quienes desde la tradición feminista descolonial advirtieron sobre el carácter colonial de la propia categoría de género (LUGONES, 2005; ESPINOSA, 2013). Crítica del devenir de la "perspectiva de género", León (2004, pág. 30) señala que junto con las alternativas teóricas y metodológicas que aportó la noción de género, surgieron consecuencias no esperadas y destaca entre ellas que: "el contenido subversivo de ciertas demandas se neutraliza y las relaciones de género comienzan a desvincularse de su partera original, la teoría feminista". Ciriza (2007) contribuye a este análisis, en un artículo que realiza una crítica feminista a "los atolladeros del género", señalando que el proceso de institucionalización de los estudios de género en universidades y centros de investigación se encuentra unido a su transnacionalización y academización, lo que impone la creciente dependencia de nuestros debates a "la moda" vigente en la academia norteamericana. Estos hechos, para la autora, enfrentan a los estudios académicos ligados al feminismo con el riesgo de una sutil domesticación.

En suma, este breve recorrido por algunas revisiones y críticas al concepto de género evidencia algunas de las razones por las cuales resulta dificil pensar en un cuerpo conceptual unificado cuando se hace referencia a los estudios de género y feministas. Más bien lo que refleja es la concurrencia de tradiciones diversas, antagonismos teóricos y políticos, ubicaciones desiguales y relaciones de hegemonía/subalternidad de acuerdo a la procedencia de las autoras. En ese sentido, la amplia difusión de la noción de género no es una condición suficiente para constituir un campo único de saber. Al respecto vuelven a resultar útiles las reflexiones de Ciriza (2007, pág. 35) cuando precisa: "Los estudios de género 
incluyen los estudios de la mujer, pero no exclusivamente. No se trata, por lo tanto, sólo de una ampliación o una complejización de la problemática, sino de la inclusión de nuevas perspectivas. Sin embargo sería curioso concluir de ello la complementariedad o continuidad de las miradas teóricas, o tan siquiera la comunidad de intereses y objetivos políticos. En todo caso tales continuidades o discontinuidades dependerán mucho más de la especificidad de las genealogías teóricas y políticas y de las tradiciones disciplinares que de cualquier suerte de afinidad nacida del uso, pretendidamente común, de la noción de género como espacio de comunicación universal y de superación del obstáculo biologicista".

\section{Algunas consideraciones sobre el concepto "salud"}

El presente apartado recoge algunas consideraciones generales acerca del campo de la salud. Nuestro propósito es mostrar que en él confluyen múltiples miradas y perspectivas, en las que no indagaremos profundamente, que complejizan aún más el universo temático del género y la salud como problemática.

Para intentar una reflexión sobre el concepto de salud es preciso indagar en el contexto histórico en que dicho concepto es producido. Esto permite comprender más cabalmente qué necesidad político-social subyace al mismo. "En su calidad de representación social, la noción de salud se ha ajustado a los modelos económicos y políticos predominantes en cada momento histórico" (ARIAS, 2013, pág. 23).

Canguilhem (2004) intenta sintetizar críticamente los puntos de vista de algunos pensadores de la llustración y hace un planteo que resulta útil para alimentar el debate: "Salud no es un concepto científico, es un concepto vulgar" (CANGUILHEM, 2004, p. 52). En este sentido, lo que suele aparecer en escritos filosóficos y tratados de medicina, refiere al "correcto funcionamiento" de los mecanismos del cuerpo, poniendo el foco en los desajustes de dicho mecanismo. Es decir, no era la salud el núcleo de las preocupaciones, sino los desarreglos del organismo que podrían constituir "la enfermedad".

La preocupación por la salud o, más precisamente, por el correcto funcionamiento del cuerpo humano desde un punto de vista científico, es propia de la modernidad capitalista. La medicina moderna tiene un lugar central en el proceso de consolidación y reproducción del sistema capitalista y en las intervenciones de éste sobre los cuerpos. Tanto la especificidad de su objeto de estudio como la innovación en sus técnicas, permitió el acceso a los cuerpos humanos con una precisión que no ha dejado de crecer a lo largo de estos siglos. Pero la práctica médica no acotó sus límites a los mecanismos del cuerpo, sino que desplegó sus discursos "de verdad" en innumerables reductos de la vida cotidiana que antes no revestían mayor interés social y, mucho menos, científico.

La consolidación de la moderna medicina como disciplina científica prepon- 
derante, poseedora no sólo de "la verdad" acerca de los cuerpos y sus procesos, sino también acerca de las prácticas correctas o incorrectas, normales o desviadas, coincide con el proceso histórico de consolidación del capitalismo como sistema dominante (FOUCAULT, 2008). Esta visión se tradujo en un modelo específico de atención, orientado a atacar las causas biológicas o fisiológicas de la enfermedad. El cuerpo entendido como máquina está hecho de piezas con funcionamiento relativamente independiente, lo que permite que cuando una de las piezas (o su mecánica) se descompone pueda ser tratada específicamente $o$, llegado el caso, extraída si su función no es considerada "vital".

Con el paso del tiempo, aparecen distintos enfoques, que modifican no sólo esta visión mecanicista del cuerpo, sino también cómo atender el binomio salud-enfermedad. Siguiendo a Rubén Gómez Arias, podemos diferenciar dos grandes paradigmas: el bienestarista y el liberal. El primero surge en el contexto de posguerra, regido por un principio de bienestar. "El interés de los estados keynesianos por el bienestar no sólo impulsó alrededor del mundo una noción más integral de salud, que intentaba superar la ausencia de enfermedad, sino también un sistema de prestación de servicios estatales dirigidos a controlar los determinantes ambientales, culturales y económicos del bienestar de la gente" (ARIAS, 2013, p. 23). En este momento se crea la Organización Mundial de la Salud, cuya definición de salud y lineamientos dan cuenta del horizonte del momento. "La salud es un estado de completo bienestar físico, mental y social, y no solamente la ausencia de afecciones o enfermedades." (ORGANIZACIÓN MUNDIAL DE LA SALUD, 2006).

El segundo paradigma abordado por Gómez Arias se refiere a la salud en las economías liberales: "En primer lugar, la salud es considerada por los economistas liberales como un asunto individual que las personas deben resolver por si mismas" (ARIAS, 2013, p. 24). Su definición de salud se refiere a la ausencia de enfermedad y las intervenciones propuestas para la atención se reducen a servicios médicos curativos. Aquí reaparece, con distintos tintes, la definición biologicista presente en los inicios de la medicina moderna.

Finalmente, es posible identificar un paradigma alternativo a los presentados anteriormente: el pensamiento contrahegemónico, cuyas corrientes más visibles han sido la Medicina Social y la Salud Colectiva. Este tradición teórica y política se ordena sobre dos pilares fundamentales: concebir la salud como un derecho humano y social fundamental, que no puede ser sometido a la lógica del mercado, sino que debe ser garantizado por el Estado de manera universal; y la comprensión de la determinación social de la salud y la enfermedad, distanciándose de las miradas biologicistas propias del pensamiento hegemónico en salud (ISTÚRIZ, ACEVEDO e JIMÉNEZ, 2012). Se trata de una corriente contrahegemónica que plantea una definición de salud que permite abordar críticamente tanto las políticas sanitarias como la atención, al mover el foco de interés de los procesos fisiológicos a los procesos sociales para comprender cómo vivimos, de qué enfermamos y cómo 
morimos. Las conductas individuales no agotan las explicaciones acerca de los padecimientos, hay un universo social y político a tener en cuenta para acercarse más acertada y humanamente a los procesos de salud-enfermedad subjetivos.

Este somero recorrido no pretende exhaustividad, sino sintetizar algunos debates que se han suscitado acerca del concepto de salud desde diversas disciplinas. Asomarnos a la riqueza de este universo de trabajos permite entrever otro nudo complejo en el ya de por sí complejo entramado de estudios sobre género y salud, objeto del presente artículo.

\section{Estudios de género y feministas en el campo de la salud}

La salud, la enfermedad y los cuidados constituyen aspectos problematizados por la teoría feminista y los estudios de género desde sus primeros pasos. Los primeros diálogos entre los estudios de género y feministas y el campo de la salud se remontan a la segunda mitad del siglo XX en el contexto de la academia anglosajona y estadounidense; siendo este proceso en América Latina coincidente con la recuperación de la democracia en la mayoría de sus países. Sin embargo es posible rastrear como antecedente histórico, al menos para el contexto europeo, investigaciones sobre las prácticas de las mujeres médicas y sanitarias en el último tercio del siglo XIX, momento en que las mujeres empezaron a acceder a la profesión médica (GÓMEZ, 2004).

Dicha tradición académica, junto con la práctica política de los movimientos por la salud de las mujeres de los años setenta, abrieron el campo a una vasta producción de saberes críticos hacia el sexismo en la medicina y el control biomédico de los cuerpos femeninos, al tiempo que señalaron las profundas huellas que las desigualdades sexo-genéricas imprimen en la salud y el bienestar de las mujeres y propusieron alternativas para abordajes sanitarios que promuevan la tratos igualitarios entre varones y mujeres. La recuperación histórica del rol protagónico de sanadoras, curanderas y cuidadoras en las familias y comunidades ha sido otro de los aportes sustanciales con los que la teoría feminista ha enriquecido, y puesto en tensión, el conocimiento acerca de la salud humana.

Como ya señalamos, en la actualidad resulta extensa y múltiple la bibliografía que analiza desde distintas perspectivas el modo en que las desigualdades sexo-genéricas operan en la salud, la enfermedad y las formas de atención, y sus aportes recorren desde las ciencias biomédicas hasta las sociales (ESTEBAN, 2006). Para aproximarnos a una definición de este campo recuperamos lo que propone Nogueiras (2013, pág. 5) cuando señala los diversos intereses por los que discurre la teoría feminista en las investigaciones sobre salud/enfermedad: "En la teoría feminista, el malestar, la enfermedad y el deterioro de la salud de las mujeres se relacionan estrechamente con la imposición de los mandatos y roles tradicionales de género, la dedicación al cuidado de otras personas y la postergación del propio, 
la sobrecarga física y emocional derivada de la doble y triple jornada, la desvalorización personal y social, el control, el uso y abuso de la sexualidad femenina, la maternidad obligatoria y sin corresponsabilidad social, las dificultades para acceder y permanecer en el empleo, la precariedad laboral, la feminización de la pobreza, la violencia estructural y la violencia directa."

Esta multiplicidad de temáticas, que responden a fenómenos de diversas escalas y que a la vez resultan interdependientes, nos conduce a identificar los aportes más relevantes y a proponer un agrupamiento posible, en ambos casos se trata de procedimientos de selección y clasificación de carácter situado. Es decir, se trata de una categorización que surge a partir de nuestra experiencia en el campo temático de la salud de las mujeres y los estudios feministas y de género. Esta experiencia se vincula con nuestros recorridos biográficos, académicos y profesionales: especialmente con el trabajo que hemos desempeñado en hospitales (como socióloga y trabajadora social), con los caminos que han surcado nuestras tesis doctorales y con algunas experiencias de docencia sobre el tema en ámbitos sanitarios y académicos. Por ese motivo, se trata de una clasificación sui generis, que no pretende ser exhaustiva, sino reunir nudos problemáticos identificados como tales a partir de las trayectorias mencionadas.

Cada categoría está integrada por una profusión de textos y por una diversidad de origenes disciplinares de las autoras. Hemos abordado trabajos provenientes de las ciencias de la salud (medicina, enfermería y epidemiología), otros inscriptos en las ciencias sociales (especialmente sociología y antropología), también incluimos aportes desde la psicología, la historia, y el derecho, entre otros. Esta conjunción de diferentes disciplinas es atribuible al propio carácter multidisciplinario de los estudios de género y feministas. La revisión incorpora autoras de procedencias diversas; muchas de ellas escriben desde América Latina, otras desde Europa (España e Italia principalmente) y algunas de las citadas provienen de Estados Unidos. Lo que unifica las diversidades mencionadas, permitiendo en cada caso agruparlas en una categoría determinada, es una problemática central por la que discurren los aportes: la intervención biomédica sobre el cuerpo de las mujeres; el androcentrismo y los sesgos de género en medicina; las enfermedades y padecimientos como experiencias marcadas sexualmente $y$, finalmente, la preocupación por la salud sexual y reproductiva de las mujeres. Entendemos que cada uno de estos tópicos se constituye en nudos conceptuales que permitirán, antes que una mirada exhaustiva, una aproximación a los debates nodales entre feminismos, estudios de género y campo de la salud.

\section{a. Intervención biomédica sobre el cuerpo de las mujeres}

La presente sección reúne estudios que analizan el avance de la medicina sobre la vida de las mujeres, transformando aspectos de la cotidianeidad en objetos 
de la ciencia médica. Este proceso tiene como finalidad el ejercicio de un control social que garantice una cierta adecuación a la norma (VIEIRA, 2008). Muchas autoras conceptualizan este fenómeno como medicalización, término acuñado y ampliamente utilizado fuera de los feminismos y los estudios de género. Sin embargo, existen otras autoras que lo abordan sin utilizar esta categoría teórica. Este apartado toma en consideración esta variedad de aproximaciones a la temática.

Los estudios agrupados en esta línea, aun cuando reconocen la medicalización como una estrategia general de control social, sostienen que el caso de las mujeres no constituye un ejemplo más sino una situación paradigmática (ESTEBAN, 2003). Efectivamente, múltiples investigaciones provenientes de distintas disciplinas han constatado la mayor coerción ejercida por las instituciones médicas sobre los cuerpos femeninos, que resultan etiquetados como enfermos más fácilmente que los masculinos (ANDREANI, 1998; GÓMEZ, 1999; RUIZ, 2011; NIEVES, 2012).

Numerosas autoras reconstruyen las condiciones históricas de surgimiento de la ciencia médica y su papel central en la colonización del cuerpo de las mujeres, proceso que se inició en los albores del capitalismo. Este sistema, que se impuso a través de la violencia como respuesta de las clases dominantes a la crisis del sistema feudal, asignó valores y roles específicos a los cuerpos humanos. La asignación diferencial de funciones a varones y mujeres está anclada en una asignación diferencial de valor a sus cuerpos. Mientras que los cuerpos de los varones se transformaron en máquinas de trabajo para satisfacer las necesidades del sistema capitalista, los cuerpos de las mujeres se convirtieron en productores de mano de obra. En simultáneo a la aparición de gran cantidad de normas y regulaciones que los incipientes Estados pusieron en marcha apoyados en y por la biomedicina, se dio un proceso de masculinización del saber sobre los cuerpos y sus procesos a partir del cual las mujeres fueron expulsadas de las actividades relacionadas con el ejercicio de la medicina y el parto. Los saberes acumulados durante siglos por ellas acerca de sus cuerpos, su sexualidad, la anticoncepción, el embarazo, el parto también fueron considerados peligrosos y perseguidos por estar relacionados con la brujería y los "delitos reproductivos" como el aborto y el infanticidio (FEDERICI, 2010; EHRENREICH e ENGLISH, 1973).

Otras investigaciones, vinculadas a la historiografía social y la historia de la medicina, realizan una deconstrucción histórica de la producción de saber y verdad en los discursos médicos sobre las mujeres (SÁNCHEZ, 2008). Indican que desde el Renacimiento, cuando se consolida el mito que reduce a la mujer a sus órganos reproductivos (ANDREANI, 1998), la medicina ha justificado las desigualdades sociales entre varones y mujeres, a través de las diferencias anatómicas, fisiológicas y patológicas de los sexos (RUIZ, 2011). Señalan una persistencia en los discursos médicos de diferentes períodos históricos, que busca explicar la subordinación de las mujeres por su naturaleza (ANDREANI, 1998; SÁNCHEZ, 2008) mediante su consideración como meras reproductoras biológicas. Ello ha 
implicado la supervisibilización de sus roles reproductivos y la invisibilización de su aportación a la economía, la política y la religión (ESTEBAN, 2006). Gran parte de estos discursos médicos de fines del siglo XIX y principios del siglo XX, contribuyeron a crear una imagen de la vida de las mujeres como una sucesión de crisis temibles debido a los ciclos reproductivos; junto con una idea de una constitución femenina naturalmente débil y enferma, pobre y susceptible mentalmente. Todo ello sirvió de sustento a diversas estrategias de control social, entre ellas, la reclusión de las mujeres en el ámbito privado.

Un claro ejemplo lo constituyen las primeras teorías de la psiquiatría, que atribuían los problemas mentales que pudieran sufrir las mujeres a sus características biológicas para la reproducción; consecuentemente, sostenían la existencia de una mayor susceptibilidad femenina para enfermar mentalmente debido a la relación entre su sistema nervioso y sus órganos reproductivos (ANDREANI, 1998; GÓMEZ, 1999; RUIZ, 2011; NIEVES, 2012). Estas creencias tenían su correlato en prácticas terapéuticas diferenciales, por lo que fue habitual la exploración de los genitales para diagnosticar cualquier enfermedad, como también la indicación de extirpar los órganos reproductivos femeninos, suponiendo que en ellos radicaba la causa del trastorno mental (GÓMEZ, 1999; CANTERO e VERDÚ-DELGADO, 2004; BERNABEU-MESTRE, ET AL., 2008).

En América Latina, y particularmente en Argentina, la injerencia creciente de la medicina en la vida cotidiana se dio a finales del siglo XIX y principios del $X X$, de la mano de los preceptos higienistas. Los Estados y diversas instituciones religiosas y civiles desplegaron una serie de estrategias no sólo de atención, sino también pedagógicas y normativas respecto de la maternidad, la salud de la prole, los cuidados correctos, la economía doméstica y los criterios de higiene y alimentación a seguir, cuyo principal objetivo fueron las mujeres en tanto madres y reproductoras de las nuevas generaciones de habitantes de la Nación (NARI, 2004; DILISCIA, 2005).

\section{b. Estudios sobre androcentrismo y sesgos de género en la biomedicina}

En El Segundo Sexo, libro de carácter fundacional para el pensamiento feminista, Simone de Beauvoir (1965 [1949], pág. 12) exponía su tesis fundamental: "[...] así como para los antiguos había una vertical absoluta respecto a la cual se definía lo oblicuo, hay un tipo humano absoluto que es el masculino [...] La humanidad es macho, y el hombre define a la mujer no en sí, sino respecto de él; no la considera como un ser autónomo [...] La mujer se determina y diferencia con relación al hombre, y no éste con relación a ella; ésta es lo inesencial frente a lo esencial. Él es el sujeto, él es lo Absoluto: ella es el Otro". Si bien Simone de Beauvoir no se refirió a este fenómeno en términos de androcentrismo, sin duda la tesis que recorre su ensayo, las mujeres como lo Otro o lo inesencial respecto de los 
varones, resultó el sustento de producciones feministas posteriores sobre el tema.

Particularmente desde el terreno de las epistemologías feministas el problema del androcentrismo ha sido ampliamente abordado, a partir de distintas tradiciones (HARAWAY, 1991; KELLER, 1991). De modo sintético, podemos decir que uno de los planteos centrales de esta crítica es que, si bien la ciencia se presenta como universal, se construyó a partir de la experiencia de una parte privilegiada de la humanidad: algunos sujetos masculinos (VALDIVIESO, 2007). Bajo la apariencia de presunta universalidad, la ciencia moderna sólo ha representado a los sectores dominantes encarnados en el varón-blanco-burgués-capaz, relegando al lugar de otredad a las y los sujetos negados por este discurso (LEÓN, 2011).

Las ideas médicas han sido objeto de esta crítica de una manera muy precisa. Al examinar el androcentrismo en la medicina Valls-Llobet (2009) remarca que ser mujer no es sólo no ser hombre. Destaca que las distintas especialidades médicas aun cuentan con pocos datos de investigación diferenciados por sexo, lo que contribuye a homogeneizar los cuerpos de varones y mujeres, bajo la preeminencia del cuerpo masculino como modelo. Para la autora, el androcentrismo biomédico se manifiesta de diversas maneras: en el hecho de que las mujeres sean invisibles para la atención sanitaria, el diagnóstico y el tratamiento de muchas enfermedades; pero también en la frecuencia con que los síntomas que padecen son confundidos, minimizados e incluso erróneamente diagnosticados.

Una línea de estudios que se desprende de estos cuestionamientos es aquella que alerta sobre la invisibilización de las mujeres en la investigación biomédica y farmacológica, preocupación que se hace presente en la bibliografía científica desde principios de 1990 (CANTERO e VERDÚ-DELGADO, 2004). Dicha invisibilización se manifiesta en la exclusión sistemática de mujeres en investigaciones biomédicas y en la aplicación mecánica y directa de los resultados obtenidos en varones a ellas (AZNAR, 2008; VALLS-LLOBET, 2009; GAVIRIA e ALARCÓN, 2010; GALAVERNA, 2010; RUIZ, 2011). Tales características de la investigación biomédica han sido conceptualizadas por algunos/as autores/as en términos de sesgo de género (CANTERO e VERDÚ-DELGADO, 2004) que consistiría en considerar que más allá del aspecto reproductivo, las diferencias en la salud-enfermedad entre varones y mujeres no existen o son irrelevantes (CERÓN-MIRELES et al., 2006).

Las autoras que investigan en esta línea sostienen que la exclusión de las mujeres en los ensayos biomédicos y farmacológicos es explicada en términos sexistas, por las potenciales "distorsiones" que introducirían los ciclos hormonales en la recolección y procesamiento de datos de investigación (GAVIRIA e ALARCÓN, 2010; GALAVERNA, 2010; RUIZ, 2011). Asimismo, señalan que en las ocasiones en que incluyen a mujeres los datos no se analizan por sexo y se asume que no hay diferencias fisiológicas relevantes con los hombres (GAVIRIA e ALARCÓN, 2010). Como consecuencia se conocen mucho menos los procesos fisiológicos de 
las mujeres que los de los hombres (SEN e ÖSTLIN, 2005, pág. 28).

Los estudios basados en modelos masculinos y sin pruebas convincentes de aplicabilidad a las mujeres, generan impactos en la práctica clínica. En efecto, producto de investigaciones que excluyen de manera sistemática las problemáticas y las experiencias de las mujeres, que las consideran apéndices o variaciones de la norma masculina o las reducen al significado femenino heteropatriarcal, las respuestas que dadas por biomedicina suelen no ajustarse a las necesidades específicas de las mujeres (SEN et al., 2005; TASA-VINYALS et al., 2015). Algunas de las consecuencias más habituales de los mencionados sesgos incluyen desde errores médicos, fallas o demoras diagnósticas, subtratamientos, subregistros y medicalización (GALAVERNA, 2010).

Ahora bien, la investigación biomédica no sólo se encuentra atravesada por el androcentrismo y los sesgos de género. El racismo y la exclusión de poblaciones racializadas resulta un rasgo sobre el que diversos estudios han llamado la atención (SEN et al, 2005; OLIVEIRA, 2006; GAVIRIA \& ALARCÓN, 2010). Esta exclusión no solo se basa en concepciones discriminatorias, sino también en intereses mercantiles de los laboratorios que financian gran cantidad de investigaciones médicas. Al respecto Oliveira (2006, pág. 76) señala: "la industria farmacéutica [...] no se interesa por fabricar medicamentos para las enfermedades prevalentes en las poblaciones pobres, dentro de las cuales no es posible no incluir a los negros". La autora afirma que las mujeres de color y pobres no solo han sido históricamente excluidas de las investigaciones en salud, sino que en las ocasiones en que se ha propiciado su inclusión ha sido en condiciones abusivas, discriminatorias e inhumanas. Documenta casos de utilización de prisioneros/as políticos y de lactantes seronegativos en el Congo como "conejillos de Indias" para probar vacunas experimentales. Además denuncia que tales investigaciones niegan sistemáticamente la continuidad del tratamiento a las personas participantes una vez terminada la investigación.

\section{c. Construcciones sexuadas de enfermedades y padecimientos}

Los estos estudios que reunimos en esta línea de investigación, analizan la forma en que factores sociales y condiciones de vida afectan la salud. En el contexto de sociedades patriarcales, es habitual que las mujeres enfrenten experiencias de discriminación; violencia física, psicológica y/o simbólica; dificultades para acceder en condiciones de igualdad al mercado de trabajo; doble o triple jornada laboral; sobrecarga de actividades de cuidado; desigualdad en el acceso a sus derechos y a la justicia; limitaciones para decidir sobre su propio cuerpo; entre otros factores que van modelando formas específicas de enfermedad y padecimiento. Así, esta perspectiva se preocupa por las experiencias, situaciones, contextos que llevan a enfermar de modos diferenciales a mujeres y varones, como también a 
desarrollar con mayor prevalencia cierto tipo de padecimientos.

El supuesto general sostenido por estas perspectivas es que la salud de hombres y mujeres es diferente y también desigual "diferente porque existen factores de tipo biológico y fisiológico que tienen distintas implicaciones en su salud; desigual porque hay factores sociales que establecen diferencias evitables, y por ello injustas, en la salud de ambos sexos" (CANTERO, 2011, pág. 11). Se visualiza al sexo-género como un determinante en el proceso de salud/enfermedad/ atención, que opera en forma conjunta con otros factores (clase, etnia, edad). Estos estudios enriquecen las explicaciones sobre la morbi-mortalidad al indicar que ésta no sólo se sustenta en el nivel biológico, sino que también intervienen los factores socioculturales y psicológicos (CARDACl, 2006). Complementariamente indican que la ubicación de las mujeres en posiciones de subordinación, en el marco de sociedades patriarcales, supone su desempoderamiento y falta de recursos materiales y simbólicos, lo que produce formas genéricamente diferenciadas de salud y enfermedad (ANDREANI, 1998; BURÍN, 1990; KRIEGER, 2003; MARKEZ ET AL., 2004; RUIZ, 2011).

Algunas de las contribuciones clásicas al respecto provienen del terreno de la epidemiología. Tal es el caso de los aportes que Krieger (2003) realiza desde la perspectiva epidemiológica ecosocial. En uno de sus artículos (2003) la autora analiza diversas expresiones de relaciones de género como la segregación sexual de la fuerza de trabajo, la discriminación en los salarios, las expectativas diferenciales sobre conducta sexual y la violencia de género, entre otros aspectos, que enlaza con especificidades corporales y biológicas de las mujeres para explicar la mayor prevalencia, o los diferentes riesgos de exposición, a enfermedades como SIDA, cáncer, cardiopatías y accidentes laborales. La misma autora resultó pionera junto con Elizabeth Fee (KRIEGER \& FEE, 1994) en considerar conjuntamente las desigualdades sexo-genéricas, raciales y de clase que enfrentaban mujeres de distintos grupos sociales en Estados Unidos y su vinculación con diferencias en la prevalencia, acceso a tratamientos y evolución de ciertas enfermedades, entre las que destacaban el SIDA. Las autoras (1994, pág. 275) afirmaban: "Las mujeres y los hombres somos diferentes, pero también tenemos similitudes, y estamos divididos por relaciones sociales de clase y raza/etnia. Para comenzar a entender cómo nuestra organización social afecta nuestra salud, debemos preguntar, repetidamente, qué es diferente y qué es similar a través de las desigualdades sociales de género, color y clase. No podemos suponer que solo la biología proporcionará las respuestas que necesitamos; en cambio, debemos replantear los problemas en el contexto de la configuración social de nuestras vidas humanas, como criaturas biológicas y actores históricos".

Dentro de este campo, nos interesa destacar un área temática que ha tenido un significativo desarrollo: la de la salud mental de las mujeres. Las primeras investigaciones sobre este tema se realizaron en Estados Unidos por la década del 
setenta preocupadas por la sobrerrepresentación de mujeres en las estadísticas psiquiátricas, como también en hospitalizaciones, uso de servicios ambulatorios y prescripción de psicofármacos (SACRISTÁN, 2009). Entre los hallazgos de esta prolífica línea de investigación, un grupo de trabajos se ocupa de exponer cómo los roles tradicionalmente asignados a las mujeres como esposas, madres y amas de casa se caracterizan por requerir de altruismo, servilismo, capacidad de contención emocional, inhibición de la agresividad, entre otros, lo que crea condiciones de vida perjudiciales para su salud mental (BURÍN, 1990). Asimismo, implican tareas carentes de estatus e invisibilizadas en cuanto a su importancia social que contribuyen a ubicar a las mujeres en posiciones de subordinación, desempoderamiento y falta de recursos materiales y simbólicos, lo que deviene en una posición crítica para preservar la salud mental (BURÍN, 1990; ANDREANI, 1998; SACRISTÁN, 2009).

Las autoras que abonan esta temática refieren también a los estereotipos reinantes de feminidad en nuestras sociedades, como factores de riesgo para la salud mental de las mujeres. Características como la autodesvalorización, la represión de los impulsos activos fuera del campo de la vida privada, la dependencia, la autoculpabilización, la supuesta debilidad y la represión de la ira, se imponen socialmente como rasgos típicos de la subjetividad femenina e implican mayor vulnerabilidad frente a la depresión (DIO BLEICHMAR, 1992; AZNAR, 2008). Asimismo destacan como factor de riesgo para el desarrollo posterior de cuadros depresivos las situaciones de violencia física, simbólica y sexual a las que se ven expuestas en forma cotidiana las mujeres (AZNAR, 2008). Justamente, en la actualidad, la patología depresiva constituye el malestar psíquico y la causa de discapacidad más frecuente en las mujeres, con una prevalencia femenina de dos por uno en relación con los varones (VALLS-LLOBET, 2009).

\section{d. Salud sexual y reproductiva y derechos.}

Esta última categoría reúne trabajos que se ocupan de la Salud Sexual y Reproductiva (SSyR) de las mujeres. Es una temática amplia que traspasa los límites del campo de la salud y se vincula con cuestiones de ciudadanía de mujeres y derechos humanos. Sucede que el mismo concepto de SSyR se reconoce a la luz de las luchas feministas y de mujeres por el reconocimiento y la garantía de los Derechos Sexuales y Reproductivos (DDSSyRR). Consideramos importante abordar este abanico de problemáticas por la importancia política que revisten en los contextos latinoamericanos en general, $y$ argentino en particular. Si bien existen diferencias y matices entre los países de la región, podemos identificar que el derrotero del reconocimiento y acceso a los DDSSyRR ha sido fragmentado e inconcluso.

La configuración de la salud y la sexualidad como derechos es la cristalización de las luchas sociales para hacer patente el entrecruzamiento de las esferas 
pública y privada, así como también las regulaciones a las que los cuerpos de los/as ciudadanos/as han sido y son sujetados: el nacer, el morir, la cantidad de hijos/as, las sexualidades, no son un asunto puramente individual y privado, sino cuestiones sociales que los Estados modernos regulan.

El derecho a la planificación familiar aparece contemplado en el documento de la Convención sobre la Eliminación de todas las formas de Discriminación contra la Mujer, específicamente ratificado en las Conferencias de El Cairo y Beijing, celebradas en 1994 y 1995, respectivamente. En dichas conferencias “(...) se establecen las bases para lograr la vigencia de los derechos reproductivos de las mujeres, y donde se incorpora la perspectiva de la equidad en la relación entre los sexos, es decir, se insta a los gobiernos a considerar en las políticas públicas, la perspectiva de género" (MENG, 2006).

El reconocimiento por parte de los organismos internacionales de la sexualidad y la reproducción como derechos humanos de las mujeres fue el resultado de años de trabajo realizado por las activistas feministas, que urdieron estrategias de acompañamiento mutuo y autoconocimiento. La obra "Nuestros cuerpos, nuestras vidas", elaborado por The Boston women's health book collective (1972) condensa esta tarea emprendida por las feministas y sienta las bases de muchos de los temas que aún hoy siguen siendo objeto de discusión y producción teórica y científica.

Existen numerosos trabajos de autoras/es de América del Sur que trazan el recorrido del acceso a los DDSSyRR de las mujeres. Abordan un amplio abanico de temas, desde la regulación de la sexualidad, pasando por el estado de situación de la SSyR, hasta las experiencias de aborto (MÓRAN FAÚNDES et al., 2012; BROWN, 2006; ABRACINSKAS \& GÓMEZ, 2007; LANGER, 2002; ERVITI, CASTRO \& SÁNCHEZ, 2006). En términos generales, y más allá de la variedad de enfoques teórico-metodológicos, los estudios citados dan cuenta de la distancia entre reconocimiento y acceso real a los DDSSyRR. Brown explora los límites entre lo público y lo privado para ofrecer una reflexión sobre el proceso de visibilización de los DDSSyRR. En el mismo campo de temas, el libro editado por Morán Faúndes et al., se aboca a temas de sexualidad y derechos y establece una agenda de DDSSyRR. El trabajo coordinado por Petracci repasa los debates alrededor de los DDSSyRR en el contexto argentino. Por su parte, el libro coordinado por Abracinskas y López Gómez reconstruye el debate acerca de la legalización del aborto en Uruguay, lo que implica un fructífero recorrido por las instancias de debate de la Ley de Salud Reproductiva en dicho país. El artículo de Erviti, Castro y Sosa Sánchez también aborda la cuestión del aborto, pero a través de la exploración de las miradas de los profesionales médicos en torno al tema, resaltando la existencia de estereotipos de género y otros marcos clasificatorios que se ponen en juego en la atención de las mujeres. El texto de Langer se ocupa del embarazo no deseado y sus consecuencias para las mujeres y para el sistema sanitario, puesto que su correlato inmediato es el aborto en condiciones 
inseguras en regiones donde esta práctica continúa siendo ilegal.

Nos resulta fundamental resaltar la vigencia de este núcleo temático en estas latitudes. Si bien en término generales coincidimos con el planteo de Esteban (2006) respecto de las limitaciones de hacer análisis reproductivistas de los cuerpos de las mujeres, es innegable que en países en los que no está garantizada la educación sexual, el acceso a información sobre SSyR y métodos anticonceptivos y el derecho a la interrupción voluntaria del embarazo, aparece como urgente la necesidad de dar cuenta científica y teóricamente de este campo de problemas.

\section{Recapitulación y reflexiones finales}

La producción de intelectuales feministas y de activistas sobre problemáticas de género en torno a la salud de las mujeres tiene casi medio siglo de existencia y es conocimiento situado. Se trata de saberes elaborados en distintas partes del mundo, anclados en tradiciones teóricas diversas y que constituyen un corpus de conocimientos amplio, variado y disímil en cuanto a perspectivas, puntos de interés y tipo de aportes realizados. Esta diversidad se explica, entre otras cosas, por las diferentes formas que han asumido las relaciones entre el movimiento de mujeres/feminista y la academia en paises del norte y del sur global, que se traduce en diferentes posiciones teórico-políticas de las investigadoras. Al mismo tiempo, las preocupaciones y temáticas abordadas responden a la coyuntura económica, política y social de los diferentes países y regiones. En ese sentido, en palabras de Rich (1999, pág. 33) debemos comprender "cómo un lugar en el mapa es también un lugar en la historia" dentro del cual somos creadas y tratamos de crear.

Al inicio del documento presentamos sucintamente los problemas identificados para realizar un recuento y elaborar una categorización de un campo tan amplio y variado, explicando que por ello no pretendiamos exhaustividad, sino destacar y recuperar el largo camino recorrido por los feminismos en el campo de la salud. Sin embargo, queremos señalar que también las dificultades para lograr exhaustividad en la revisión de investigaciones feministas/de género sobre salud puede asociarse, al menos en parte, al lugar marginal que estas producciones tienen dentro del universo académico y de los ámbitos de circulación del mainstream científico. Marginalidad que se acrecienta cuando de trabajos de América Latina se trata. Así, rastrear y acceder a publicaciones feministas supone el trabajo de reconstruir una trama cuyos hilos se encuentran dispersos, en gran medida, porque "las relaciones con quienes nos antecedieron en la crítica del orden patriarcal son discontinuas" (CIRIZA, 2015, pág. 85). En ese contexto, y situadas en Argentina, nos resulta relevante incluir las producciones teóricas locales/regionales junto con los trabajos europeos o estadounidenses que cuentan con mayor circulación y difusión.

En la revisión y categorización propuesta se hacen evidentes preocupaciones e intereses comunes y, al mismo tiempo, divergencias. Uno de los hilos que liga 
los distintos trabajos es la crítica al biologicismo como herramienta del orden establecido para justificar la subordinación de las mujeres. Este es un nudo problemático para la teoría feminista desde sus primeros pasos, que se ha desplegado en variados campos. Sin embargo, la importancia estratégica de las contribuciones feministas al campo de la salud radica en que mediante las discusiones mantenidas con la biomedicina -específicamente con disciplinas como la ginecología y la psiquiatría- han aportado sólidos argumentos para desmontar el supuesto de que las desigualdades sociales existentes entre varones y mujeres se justifican en diferencias biológicas.

El otro aspecto compartido que atraviesa las producciones reseñadas previamente radica en el vínculo con el movimiento feminista y de mujeres. Este vínculo puede ser, en algunos casos, próximo y manifiesto o más difuso e, incluso, no reconocido en otros. Lo que aquí sostenemos es que, así como el movimiento retoma argumentos teóricos/científicos elaborados en ámbitos académicos para debatir en el escenario político, las preguntas que guían las investigaciones abrevan de la agenda feminista aunque no siempre explícitamente. En este punto cabe recuperar la reflexión de Eva Rodríguez Agüero (2015) acerca del carácter complejo del intercambio de ideas entre distintos ámbitos. De modo tal que los vínculos entre producciones academicas y activismo no son lineales ni transparentes e implican una reapropiación transformadora de temáticas y enfoques. Un caso paradigmático es el del reconocimiento de la salud sexual y reproductiva como derecho humano. Esta problemática llega a la agenda pública de los organismos internacionales y los Estados a partir de la retroalimentación entre activismo e investigación.

En cuanto a las diferencias que es posible apreciar entre los trabajos analizados, una de las que más evidentes se suscita por la categoría de género y las diversas posiciones asumidas por las autoras: mientras que unas cuestionan su uso; otras lo consideran estratégico; algunas entienden que da cuenta del carácter relacional de la posición subordinada de las mujeres, brindando complejidad a los planteos; y otro grupo de autoras utiliza la noción para superar el binarismo de la categoría de sexo. Se trata, en buena medida, de los "atolladeros del género" (ClRIZA, 2007) que para la autora abarcan un nutrido espectro de problemáticas conceptuales y políticas. Una que resulta fundamental en este artículo, radica en que el uso del concepto "género", ligado en gran medida a la institucionalización de los estudios feministas, no hace referencia al sujeto político implicado, lo que ha suscitado grandes debates al interior del feminismo y de la academia separando "las feministas" y las estudiosas del género. Cabría interrogarse, entonces, sobre la pertinencia de incluir los aportes que se reconocen como feministas dentro del campo temático de género y salud. La respuesta que encontramos a este interrogante es ser fieles a su genealogía común, ya que que los llamados estudios de género se apoyan y tienen su base en producciones teóricas feministas previas 
al uso de la noción de género.

De lo anterior se desprende una segunda diferencia vinculada a cuan estructuralmente analizan las desigualdades sexo-genéricas en el campo de la salud. Esto es, si las vinculan con el sistema patriarcal como estructura general de dominación, o si las consideran producto de sesgos o malas prácticas académicas y sanitarias, subsanables mediante correcciones metodológicas. Ello se hace particularmente evidente en las diferencias que ostentan las investigaciones sobre la medicalización del cuerpo femenino como estrategia de control social y las que abordan los sesgos de género en la práctica clínica.

Una tercera divergencia que identificamos remite a los diversos contextos de producción de conocimiento de los trabajos, que se traduce en agendas de investigación con prioridades distintas. No es de extrañar que la mayoría de los estudios de sesgos de género en salud provengan de paises centrales, que ostentan una mayor concentración de recursos para la investigación biomédica. Mientras que, en América Latina, las áreas temáticas que revisten mayor centralidad se vinculan con las deudas que los Estados mantienen en relación a garantizar los derechos de las mujeres: acceso a los servicios de salud, violencia institucional, acceso a información y las consecuencias del aborto clandestino, sólo por enumerar algunas.

El recuento de puntos de acuerdo y disenso que realizamos ha buscado exponer que dentro de los vastos límites de las categorías de "género y salud" conviven en tensión distintas posiciones, intereses, disciplinas y geografías que, más allá de las divergencias enumeradas, poseen un enorme potencial de resistencia y transformación frente a la avanzada conservadora que se registra en nuestras latitudes. Bien es sabido que en contextos de ajuste y retroceso de derechos, las conquistas de las mujeres son las primeras en desmontarse. Aquí es donde cobra importancia la reconstrucción de trayectorias y genealogías críticas.

\section{Referencias}

ABRACINSKAS, L., \& LÓPEZ GÓMEZ, A. Aborto en debate. Desafíos y problemas del Uruguay democrático. Montevideo: Mujer y Salud en Uruguay, 2007.

AZNAR, M. P. Género y salud. Suma Psicológica, v. 15, n.1, p. 75-93, 2008.

ANDREANI, F. Mujer, Cultura y Malestar Psíquico. Revista Venezolana de Análisis de Coyuntura, v. IV , n. 2, p. 189-206, 1998.

ARIAS, R. D. La noción de salud, un concepto en expansión. Medicina y sociedad, p. 20-27, 2013.

BEAUVOIR, S. El segundo sexo. Buenos Aires: Ediciones Siglo Veinte, 1965. 
BELLUCCI, M. De los estudios de la mujer a los estudios de género: han recorrido un largo camino. FERNÁNDEZ, A. M. Las mujeres en la imaginación colectiva. Barcelona: Paidós, 1992.

BERNABEU-MESTRE, J., CID-SANTOS, A. P., ESPLUGUES, J. X., \& GALIANA-SÁNCHEZ, M. E. Categorías diagnósticas y género: Los ejemplos de la clorosis y la neurastenia en la medicina española contemporánea (1877-1936). Asclepio. Revista de Historia de la Medicina y de la Ciencia, v. LX, n. 1, p. 83-102, 2008.

BROWN, J. De cuando lo privado se hace público, o de cómo se construyen las políticas de sexualidad y (no) reproducción. El caso de Mendoza. En M. PETRACCI, \& S. RAMOS, Las políticas de salud y Derechos Sexuales y Reproductivos en la Argentina: aporte para comprender su historia. Buenos Aires: CEDES, 2006.

BURÍN, M. El malestar de las mujeres. La tranquilidad recetada. Buenos Aires: Paidós, 1990.

CANGUILHEM, G. (2004). Escritos sobre medicina. Buenos Aires: Amorrortu, 2004.

CARDACI, D. Ordenando Relatos. Investigaciones sobre Salud y Género en Programas Mexicanos de Estudios de la Mujer. Salud Colectiva, n. 2, p 21-34, 2006.

CANTERO, M. T., \& VERDÚ-DELGADO, M. Sesgo de género en el esfuerzo terapéutico. Gaceta Sanitaria, v. 18, n. 1, p. 118-25, 2004. BIBLIOGRAPHY VI11274.

CERÓN-MIRELES, P., SANCHEZ-CARRILLO, C., ROBLEDO-VERA, C., DEL RÍO-ZOLEZZI, A., PEDROSA-ISLAS, L., REYES-ZAPATA, I., et al. Aplicación de la perspectiva de género en artículos publicados en cuatro revistas nacionales de salud. Salud Pública de México, v. 48, n. 4, p. 332-340, 2006.

CIRIZA, A. Apuntes para una crítica feminista de los atolladeros del género. Estudios de Filosofía Práctica e Historia de las Ideas, v. 8, n. 9, p 23-41, 2007. . Construir genealogías feministas desde el Sur: encrucijadas y tensiones. MILLCAYAC-Revista Digital de Ciencias Sociales, v. II, n. 3, p. 83-104, 2015.

COLLINS, P. La política del pensamiento feminista negro. En M. NAVARRO, C. STIMPSON ¿Qué son los estudios de mujeres? Buenos Aires: Fondo de Cultura Económica, 1998.

DAVIS, A. Mujeres, raza y clase. Madrid: Akal, 2005.

DILISCIA, M. S. Dentro y fuera del hogar. Mujeres, familias y medicalización en Argentina, 1870-1940. Signos Históricos, n. 13, p. 94-119, 2005. 
DIO BLEICHMAR, E. La depresión en la mujer. Revista de la Asociación Española de Neuropsiquiatría, 11(39), 283-289, 1992.

ERVITI, J., CASTRO, R., \& SOSA SÁNCHEZ, I. Las luchas clasificatorias en torno al aborto: el caso de los medicos en hospitales públicos de México. Estudios Sociológicos, p. 637-665, 2006.

ESPINOSA, Y. El feminismo descolonial como epistemología contra-hegemónica. Fazendo Genero 10, 2013.

ESTEBAN, M. L. El Estudio de la Salud y el Género: Las Ventajas de un Enfoque Antropológico y Feminista. Salud Colectiva(2), 9-20, 2006.

El género como categoría analítica. Revisiones y aplicaciones a la salud.Cuadernos de Psiquiatría Comunitaria, 3 (1), 22-39, 2003.

FEDERICI, S. Calibán y la bruja. Mujeres, cuerpo y acumulación originaria. Buenos Aires: Tinta Limón, 2010.

FERNÁNDEZ, A. M. Los estudios de género en la universidad. En R. T. Género, Género y Universidad. Avances y desafíos(p. 7-19). Montevideo: Universidad de la República, 2003.

FOUCAULT, M. La historia de la sexualidad. La voluntad de saber. Buenos Aires: Siglo XXI Editores, 2008.

GARCÍA, Y. Acerca del género como categoría analítica. Nómadas. Revista crítca de ciencias sociales y jurídicas, n. 12, 2006.

GALAVERNA, A. La invisibilidad de las mujeres en la medicina. Foro Nacional Interdisciplinario Mujeres en Ciencia, Tecnología y Sociedad. San Carlos de Bariloche, Río Negro, Argentina, 2010.

GAVIRIA, S., \& ALARCÓN, R. Psicopatología y género: visión longitudinal e histórica a través del DSM. Revista Colombiana de Psiquiatría, 39 (3), 389404, 2010.

GÓMEZ, A. Psiquiatría y género: el naciente discurso médico- psiquiátrico en España y el estatuto social de la mujer. Revista de la Asociación Española de Neuropsiquiatría, v. XIX, n.72, p. 637-652, 1999.

GUILLAUMIN, C. Práctica de poder e idea de naturaleza. En O. C. (compiladoras), El patriarcado al desnudo. Tres feministas materialistas (p. 19-56). Buenos Aires: Brecha Lésbica, 2005 [1992]. 
HARAWAY, D. Género para un diccionario marxista: la política sexual de una palabra. En Ciencia, cyborgs y mujeres. Madrid: Ediciones Cátedra, 1991.

ISTÚRIZ, O., FEO ACEVEDO, C., \& JIMÉNEZ, P. Pensamiento contrahegemónico en salud. Revista Cubana de Salud Pública, p. 602-614, 2012.

KELLER, E. Reflexiones sobre ciencia y género. Valencia: Alfons el Magnánim, 1991.

KRIEGER, N. Genders, sexes, and health: what are the connections and why does it matter? International Journal of Epidemiology, n. 32, p. 652-657, 2003.

KRIEGER, N., \& FEE, E. Man-made medicine and women's health: the biopolitics of sex/gender and race/ethnicity. International Journal of Health Services, v. 24, n. 2, p. 265-283, 1994.

LAMAS, M. Usos, dificultades y posibilidades de la categoría género. Papeles de población, n. 21, p. 147-178, 1999.

LANGER, A. El embarazo no deseado: impacto sobre la salud y la sociedad en América latina y el Caribe. Revista Panamericana de Salud Pública, p. 192-205, 2002.

LEÓN, M. Tensiones presentes en los estudios de género. Género, mujeres y saberes, p. 23-46, 2004.

LUGONES, M. Multiculturalismo radical y feminismos de mujeres de color. Revista internacional de filosofía política, n. 25, p. 61-76, 2005.

MARKEZ, I., POO, M., ROMO, N., MENESES, C., GIL, E., \& VEGA, A. Mujeres y psicofármacos: la investigación en atención primaria. Revista de la Asociación Española de Neuropsiquiatría, n. 91, p. 37-61, 2004.

MENG, G. Ley de salud sexual y procreación responsable argentina: ¿una politica de género? En: PETRACCI, M. \& RAMOS, S. La política pública de salud y derechos sexuales y reproductivos en la Argentina: aportes para comprender su historia. Buenos Aires: UNFPA - CEDES, 2006.

MOORE, H. Antropología y feminismo. Madrid: Cátedra, 2009.

MORÁN FAÚNDES, J. M., SGRÓ RUATA, M. C., \& VAGGIONE, J. M. Sexualidades, desigualdades $y$ derechos. Reflexiones en torno a los derechos sexuales y reproductivos. Córdoba: Ciencia, Derecho y Sociedad Editorial, 2012.

NARI, M. Políticas de maternidad y maternalismo político. Buenos Aires: Biblos, 2004. 
NIEVES, O. Foucault, el poder y la psicopatologización de las mujeres: coordenadas para el debate.Teoría y crítica de la psicología(2), 148-159, 2012.

NOGUEIRAS GARCÍA, B. Discursos y prácticas feministas en el ámbito de la salud en España (1975-2013). Tesis de Mestría. Madrid: Universidad Complutense de Madrid, 2013.

OLIVEIRA, F. Condición socioeconómica, de género y raza/etnia en las investigaciones biomédicas. En: KEYEUX, G. Ética de la investigación en seres humanos y políticas de salud pública. Bogotá: UNESCO, 2006.

ORGANIZACIÓN MUNDIAL DE LA SALUD. (1 de Octubre de 2006). www. who.int. Recuperado el 14 de Enero de 2017, de http://www.who.int/governance/ eb/who_constitution_sp.pdf?ua=1

ORTIZ GÓMEZ, T. Interacciones entre salud, historia y feminismo. Una revisión historiográfica. Diálogo Filosófico, n. 59, p. 229-244, 2004.

OSBORNE, R., \& MOLINA PETIT, C. Evolución del concepto de género. EMPIRIA. Revista de metodología de ciencias sociales, v. 15, p. 147-182, 2008.

RODRÍGUEZ AGÜERO, E. Intervenir desde los márgenes: Mujeres y feminismos en la revista Crisis. Argentina 1973-1976. Nomadias, n. 19, p 105-128, 2015.

RUBIN, G. El tráfico de mujeres: notas sobre la "economía política" del sexo. Nueva Antropología, v. VIII, n. 30, p. 95-145, 1986.

RUIZ, C. Las mujeres y la enfermedad mental. Una perspectiva de género a través de la historia contemporánea. Cuadernos Kóre. Revista de historia y pensamiento de género, v. 1, n. 4, p. 208-223, 2011.

SACRISTÁN, T. Aportaciones sociológicas al estudio de la salud mental de las mujeres. Revista Mexicana de Sociologia(4), 647-674, 2009.

SÁNCHEZ, D. El discurso médico, piedra angular de la construcción de las relaciones de género en la época contemporánea. Asclepio. Revista de Historia de la Medicina y de la Ciencia, LX (1), 63-82, 2008.

SCOTT, J. El género: una categoría útil para el análisis histórico. En J. Amelang, \& M. Nash, Historia y género: Las mujeres en la Europa moderna y contemporánea (p. 23-56). Valencia: Edicions Alfons el Magnànim, 1995.

SEN, G., GEORGE, A., \& ÖSTLIN, P. Incorporar la perspectiva de género en la equidad en salud: un análisis de la investigación y las políticas. Organización Panamericana de la Salud, 2005. 
STOLLER, R. Sex and gender. Nueva York: Science House, 1968.

TASA-VINYALS, E., MORA GIRAL, M., \& RAICH-ESCURSELL, R. M. Sesgo de género en medicina: concepto y estado de la cuestión. Cuadernos de Medicina Psicosomática y Psiquiatría de Enlace(133), 14-25, 2005.

VALDIVIESO, M. Críticas desde el feminismo y el género a los patrones de conocimiento dominantes. Revista venezolana de estudios de la mujer, 12 (28), 185-202, 2007.

VALLS-LLOBET, C. Mujeres, salud y poder. Madrid: Ediciones Cátedra, 2009.

Recebido em janeiro de 2018.

Aprovado em setembro de 2018. 